\title{
Estudo comparativo entre a amputação de reto na posição clássica de Lloyd-Davies e em decúbito ventral.
}

\section{Comparative study between amputation of the rectum in the classic Lloyd-Davies position and in ventral decubitus.}

Rodrigo Barbosa Novais'; Alex Albuquerque lins Barbosa'; Priscila Marques Intelizano'; Fang Chia Bin, TCbC-SP1; Karina Dagre Magri Castro'; Fernanda Bellotti Formiga'; Thiago Silveira Manzione, TCBC-SP'; Cauê Fedrigo loyola Batista²

R E S U M O

\begin{abstract}
Objetivo: avaliar benefícios e desvantagens do posicionamento em decúbito ventral em relação ao de Lloyd-Davies, de pacientes submetidos à amputação abdominoperineal de reto. Métodos: estudo retrospectivo de 56 pacientes submetidos à amputação abdominoperineal de reto por neoplasias de reto distal e de canal anal, tratados no Hospital Central da Santa Casa de Misericórdia de São Paulo entre 2008 e 2017. Resultados: a média de idade dos pacientes foi de 63,08 anos, sendo $48,2 \%$ deles mulheres e 51,8\%, homens. Adenocarcinoma foi o tipo histológico em $94,6 \%$ dos casos e carcinoma espinocelular em 5,4\%. A posição de Lloyd-Davies foi a adotada em 66,1\% das cirurgias e a posição ventral em 33,9\%. No momento da cirurgia quatro pacientes apresentavam metástases sincrônicas: hepática (um caso), pulmonar (um caso) e hepática e pulmonar simultâneas (dois casos). Tratamento neoadjuvante foi realizado em 85,7\% dos pacientes. Complicações pós-operatórias tardias ocorreram em 13 pacientes operados na posição clássica e em um paciente operado em decúbito ventral. O tempo de sobrevida global para o grupo operado na posição clássica foi, em média, de 45,7 meses, enquanto que no grupo operado em decúbito ventral foi de 15,5 meses. Conclusão: o grupo da posição ventral apresentou menor necessidade de infusão de volume intravenoso intraoperatório e menos complicações pós-cirúrgicas tardias, enquanto que o grupo Lloyd-Davies obteve melhores tempos cirúrgicos e anestésicos. Recidiva, tempo livre de doença e sobrevida global devem ser avaliados em um tempo maior de seguimento.
\end{abstract}

Descritores: Neoplasias Intestinais. Amputação. Neoplasias Retais. Cirurgia Colorretal.

\section{INTRODUÇÃO}

$\mathrm{O}$ câncer de cólon e reto ocupa o terceiro lugar em tipos de câncer mais incidentes no Brasil em homens (5\%) e o segundo lugar em mulheres $(6,4 \%)^{1}$. O tipo histopatológico mais comum é o adenocarcinoma, perfazendo cerca de $96 \%$ dos casos. Outros tipos histológicos são neoplasias malignas raras, algumas requerendo condutas terapêuticas específicas ${ }^{2,3}$.

A anatomia singular do reto, sua localização retroperitoneal e a proximidade de órgãos urogenitais, nervos autonômicos e esfíncteres anais, torna o acesso cirúrgico relativamente complexo. Além disso, a dissecção deve ser precisa nos planos anatômicos, já que a dissecção medial para a fáscia endopélvica que reveste o mesorreto pode comprometer o paciente com recidiva local, enquanto que a dissecção lateral ao espaço anatômico avascular cursa com risco de lesão dos nervos autonômicos mistos, ocasionando impotência em homens e disfunções de bexiga em ambos os sexos ${ }^{4}$.

A cirurgia de amputação abdominoperineal (AAP) do reto é preconizada para tumores de terço inferior do reto, canal anal, tumores com invasão pélvica, metastáticos e para os associados às doenças inflamatórias intestinais ${ }^{5}$. Dentre as técnicas de AAP, a cirurgia de Miles comporta uma colostomia definitiva, fechamento do sigmoide acima do tumor, ligadura dos vasos mesentéricos inferiores, fechamento do ânus e remoção da peça pelo períneo, englobando o ânus. É uma cirurgia de grande porte e com importantes índices de complicações intraoperatórias (sangramentos e choque hipovolêmico) e pós-operatórias (abscessos perineais, infecção do trato urinário, impotência sexual e bexiga neurogênica). A mortalidade cirúrgica da AAP encontra-se entre $0 \%$ e $6,5 \%{ }^{5-7}$. O tempo perineal da AAP pode ser realizado com o paciente em posição de Lloyd-Davies ou em posição ventral. 
Quando em posição de Lloyd-Davies, a cirurgia de Miles é feita em um só tempo com duas equipes cirúrgicas concomitantes: uma no abdome e outra no períneo, de modo que o paciente permanece em decúbito dorsal com flexão das coxas sobre o abdome em $90^{\circ}$ e das pernas sobre as coxas também em $90^{\circ}$, abduzidas e apoiadas em suportes. Tal posicionamento promove menor tempo cirúrgico, com consequente recuperação pósoperatória mais rápida e evita os riscos associados à posição ventral relacionados à diminuição do débito cardíaco e compressão de estruturas corporais ${ }^{8,9}$.

Estudos recentes sugerem que a posição ventral, para a excisão do reto, apresenta vantagens, como melhor dissecção do reto, menos sangramento e menor ocorrência de perfurações iatrogênicas. Em contrapartida, aumenta o tempo cirúrgico pela necessidade de mudança de posicionamento do paciente e pela não sincronização do tempo abdominal com a excisão do reto. Mas possui benefícios no que se refere à recidiva e melhores resultados oncológicos ${ }^{10}$.

Diante desses fatos, visa-se avaliar os benefícios e desvantagens do posicionamento em decúbito ventral em relação ao de Lloyd-Davies para execução do tempo perineal na cirurgia de AAP do reto, nos pacientes operados no Hospital Central da Irmandade de Misericórdia da Santa Casa de São Paulo.

\section{MÉTODOS}

Estudo retrospectivo de pacientes submetidos à AAP de reto por adenocarcinoma de reto distal ou carcinoma espinocelular de canal anal, tratados no Hospital Central da Santa Casa de Misericórdia de São Paulo entre os anos de 2008 e 2018. Trata-se de uma análise comparativa entre as tácticas cirúrgicas existentes para realização do tempo perineal da AAP de reto: o posicionamento de Lloyd-Davies e o decúbito ventral.
Os critérios de exclusão foram: impossibilidade de obter todos os dados necessários para análise, cirurgia de amputação de reto por doenças benignas e pacientes que, por recidiva pélvica, foram submetidos apenas ao tempo perineal complementar à retossigmoidectomia prévia.

Foram analisadas as seguintes variáveis: idade, sexo, comorbidades, neoadjuvância, estadiamento patológico, diagnóstico, tempo cirúrgico, necessidade de cristaloides ou transfusão de sangue e hemoderivados no intraoperatório, tempo de internação, margem anatomopatológica circunferencial e proximal, linfonodos dissecados, estadiamento cirúrgico, complicações perioperatórias (choque, sangramento, lesão de outros órgãos, trombose venosa profunda, retenção urinária aguda), pós-operatórias precoces (infecção e deiscência de ferida operatória, coleção pélvica, suboclusão intestinal, isquemia colostomia) e tardias (hérnia paracolostômica, incontinência urinária e impotência sexual), tempo livre de doença e sobrevida global.

A análise estatística foi realizada no programa SPSS v.13.0 (statistical package for social science). Na análise descritiva foram calculadas as frequências absolutas e relativas ( $n$, percentual) para as variáveis qualitativas e as medidas resumo (média, mediana, desvio padrão, mínimo e máximo) para as variáveis quantitativas. Foram utilizados os seguintes testes: Qui-quadrado ou exato de Fisher e t-Student ou Mann-Whitney. Foi adotado um nível de significância de 5\%.

O trabalho foi aprovado previamente pelo Comitê de Ética da Instituição, tendo o parecer no 1.252 .153 .

\section{RESULTADOS}

A amostra foi composta por 56 pacientes submetidos à AAP de reto nas posições de LloydDavis ou ventral, sendo o posicionamento ventral usado apenas a partir de novembro de 2012. 
O tempo perineal foi realizado na posição clássica de Lloyd-Davies em 37 pacientes $(66,1 \%)$ e na posição ventral em 19 (33,9\%).

A análise epidemiológica da amostra evidenciou $27(48,2 \%)$ pacientes do sexo feminino e 29 (51,8\%) do masculino. A média de idade foi de 63,08 anos, com mediana de 62 anos, sendo 37 a mínima e 87 a máxima, com desvio padrão de 12,07. Na análise das comorbidades 17 (30,4\%) pacientes apresentavam hipertensão arterial sistêmica, cinco $(8,9 \%)$ diabetes mellitus, quatro $(7,1 \%)$ eram tabagistas, um $(1,8 \%)$ era ex-tabagista e cinco $(8,9 \%)$ apresentavam outras comorbidades.

O tipo histológico foi adenocarcinoma em $53(94,6 \%)$ pacientes e carcinoma espinocelular em três $(5,4 \%)$. No momento da cirurgia, um $(1,8 \%)$ paciente apresentava metástase hepática sincrônica, um $(1,8 \%)$ pulmonar e dois $(3,6 \%)$ hepática e pulmonar simultâneas. Realizaram neoadjuvância 48 (85,7\%) pacientes, em sua maioria esquema radioterapia e quimioterapia (46 pacientes), conforme protocolo do Serviço.

Na comparação entre os tempos de anestesia e tempo cirúrgico, observou-se para o grupo LloydDavies uma média de 6,3 horas. Para o grupo ventral, observou-se uma média de tempo anestésico de 7,7 horas, sendo a comparação entre os dois grupos estatisticamente significante $(p<0,001)$. Com relação ao tempo cirúrgico, o grupo Lloyd-Davies apresentou uma média de 5,28 horas, e o grupo ventral de 6,4 horas, sendo a comparação entre os dois grupos estatisticamente significante $(p=0,006)$.

A variação da taxa de hemoglobina pré e pós-operatória dos pacientes que não receberam transfusão sanguínea, no grupo Lloyd-Davies teve uma média de $2,24 \mathrm{~g} / \mathrm{dl}$. No grupo ventral a variação média foi de $2,62 \mathrm{~g} / \mathrm{dl}$, não sendo estatisticamente significante entre os dois grupos $(p=0,442)$. A variação do hematócrito pré e pós-operatório dos pacientes que não receberam transfusão sanguínea no grupo Lloyd-Davies teve uma média de 6,81\%.
O valor negativo da diferença de hematócrito foi encontrado apenas em um paciente, provavelmente devido a erro laboratorial. No grupo ventral a variação média foi de 7,54\%, não sendo estatisticamente significante entre os dois grupos $(p=0,602)$.

Com relação à transfusão sanguínea no intraoperatório, 14 pacientes do grupo Lloyd-Davies receberam uma média de 1,8 concentrados de hemácias, com mediana de 2,0. No grupo ventral, três pacientes receberam transfusão, com uma média de 3,6 concentrados. A diferença entre os dois grupos não foi estatisticamente significante $(p=0,682)$. Analisando-se a quantidade de volume recebido no intraoperatório (cristaloides e coloides), observou-se que o grupo Lloyd-Davies recebeu, em média, 4432ml. A análise foi estatisticamente significante $(p=0,021)$.

Complicações do intraoperatório foram observadas em 13 (23,2\%) pacientes, sendo 11 $(29,7 \%)$ do grupo posição Lloyd-Davies e dois $(10,5 \%)$ do grupo ventral, não sendo a diferença entre eles estatisticamente significante $(p=0,181)$ pelo teste de Fisher. Dentre essas complicações, a perfuração do reto durante a dissecção foi observada em um $(2,7 \%)$ paciente na posição de Lloyd-Davies e em um (5,3\%) paciente na posição ventral, não sendo estatisticamente significante $(p=1,0)$, pelo teste de Fisher. A lesão de uretra foi observada em apenas um $(2,7 \%)$ paciente do grupo LloydDavies e nenhum do outro grupo. Sangramento intraoperatório foi descrito para 11 pacientes, sendo dez $(27 \%)$ do grupo Lloyd-Davies e um $(5,3 \%)$ do grupo ventral $(p=0,77)$ pelo teste de Fisher.

Das complicações ocorridas durante a internação, foram observadas trombose venosa profunda de membro inferior em um $(2,7 \%)$ paciente do grupo Lloyd-Davies e retenção urinária aguda em um $(2,7 \%)$ paciente do grupo Lloyd-Davies, não sendo observadas complicações no grupo ventral.

Foram contabilizados dois óbitos durante a internação, um $(2,7 \%)$ do grupo Lloyd-Davies e um $(5,3 \%)$ do grupo ventral, com teste de Fisher 
mostrando $p=1,0$. A causa mortis foi, respectivamente, hipercalemia secundária à insuficiência renal aguda no segundo dia de pós-operatório e sepse de foco pulmonar no 11ํ dia de pós-operatório.

Quanto à duração da internação, observouse para o grupo Lloyd-Davies uma média de 6,16 dias, e para o grupo ventral uma média de 6,37 dias. Utilizando o teste de Mann-Whitney, os postos médios respectivos foram 26,51 e 32,37, não tendo significância estatística $(p=0,193)$.

Complicações pós-operatórias precoces (até 30 dias da cirurgia) foram constatadas em 15 $(26,8 \%)$ pacientes da amostra, sendo oito $(21,6 \%)$ do grupo Lloyd-Davies e sete $(36,8 \%)$ do grupo ventral, não sendo estatisticamente significante $(p=0,223)$. Dentre as complicações, foi observada infecção de ferida operatória perineal em dez pacientes, sendo seis $(16,2 \%)$ do grupo LloydDavies e quatro $(21,1 \%)$ do grupo ventral, sem significância estatística pelo teste de Fisher $(p=0,72)$. A deiscência de ferida operatória foi observada em sete pacientes, sendo três $(8,1 \%)$ do grupo LloydDavies e quatro $(21,1 \%)$ do grupo ventral, sem significância estatística $(p=0,212)$. Abscesso pélvico foi observado em um $(2,7 \%)$ paciente do grupo Lloyd-Davies apenas, sem significância estatística $(p=1,0)$. Suboclusão foi observada em dois $(10,5 \%)$ pacientes do grupo ventral apenas, sem significância estatística pelo teste de Fisher $(p=0,111)$. Isquemia de colostomia foi observada em um $(2,7 \%)$ paciente do grupo Lloyd-Davies, sem significância estatística $(p=1,0)$.

Analisando-se as complicações tardias (ocorridas após 30 dias da cirurgia), observouse sua ocorrência em 14 (25\%) pacientes, sendo $13(35,1 \%)$ do grupo Lloyd-Davies e apenas um $(5,3 \%)$ do grupo ventral, com significância estatística $(p=0,021)$. Dentre as complicações tardias, incontinência urinária foi observada em cinco $(13,5 \%)$ pacientes do grupo Lloyd-Davies e em nenhum do grupo ventral $(p=0,155)$ pelo teste de Fisher. A impotência sexual foi observada em um $(2,7 \%)$ paciente do grupo Lloyd-Davies e em nenhum do grupo ventral $(p=1,0)$. Hérnia paracolostômica foi observada em cinco $(13,5 \%)$ pacientes do grupo Lloyd-Davies apenas $(p=0,155)$ pelo teste de Fisher. Suboclusão e abdome agudo obstrutivo foram observados em três pacientes, sendo dois $(5,4 \%)$ do grupo Lloyd-Davies e um $(5,3 \%)$ do grupo ventral, com $p=1,0$.

Com relação às margens de ressecção, a proximal foi negativa em todos os casos. A margem circunferencial foi positiva em cinco casos, sendo dois $(5,4 \%)$ do grupo Lloyd-Davies e três $(15,8 \%)$ do grupo ventral, porém não estatisticamente significante $(p=0,324)$ pelo teste de Fisher.

O número de linfonodos ressecados foi analisado pelo teste Mann-Whitney. O grupo LloydDavies obteve mediana de sete linfonodos, com mínimo de zero, máximo de 25 e posto médio de 8,51. No grupo ventral, a mediana foi de 12 linfonodos, com mínimo de zero e máxima de 23, com posto médio de 11, não sendo estatisticamente significante $(p=0,193)$.

Quanto à recidiva, seis $(10,7 \%)$ pacientes apresentaram recidiva local e cinco (8,9\%) sistêmica. Dentre os pacientes com recidiva local, cinco $(13,5 \%)$ foram do grupo Lloyd-Davies e um $(5,3 \%)$ do grupo ventral $(p=0,65)$. A recidiva sistêmica foi observada em três $(8,1 \%)$ pacientes do grupo Lloyd-Davies e em dois $(10,5 \%)$ do grupo ventral $(p=1,0)$. Para análise do tempo livre de doença foi utilizado teste log-Rank, cujo resultado não foi estatisticamente significante, com $p=0,193$.

O tempo de sobrevida global para o grupo Lloyd-Davies teve uma média de 45,7 meses, mediana de 46,1, mínima de um mês, máxima de 85,2 meses e com desvio padrão de 22,9. Para o grupo ventral, a média foi de 15,5 meses, mediana de 14,2, mínima de três meses, máxima de 33,4 meses e com desvio padrão de 7,2. 


\section{DISCUSSÃO}

O presente estudo tratou de uma amostra com uma predominância de casos de neoplasia de reto do sexo masculino, traçando paralelo com as incidências norte-americanas e globais, porém ao contrário do Brasil, onde a incidência em mulheres é discretamente maior ${ }^{1,11-14}$. A média de idade ao diagnóstico da neoplasia de reto em nossa amostra foi compatível com os encontrados na literatura, cujo valor encontra-se na faixa de 63 anos para homens e 65 para mulheres ${ }^{14,15}$. As comorbidades predominantes da amostra foram hipertensão arterial, diabetes mellitus e tabagismo. Diversos estudos já relacionaram diabetes com aumento do câncer colorretal, assim como o tabagismo (principalmente para o câncer de reto) ${ }^{16-22}$. Os tipos histológicos observados condizem com estatísticas mundiais ${ }^{2,3}$.

Em relação aos aspectos técnicos da comparação entre as duas posições cirúrgicas de interesse para a amputação abdominoperineal do reto, observou-se diferença estatisticamente significante dos tempos anestésicos médios e cirúrgicos, sendo a posição de Lloyd-Davies significantemente mais rápida de execução nos dois quesitos. Nossos dados foram contrários a outros estudos da literatura internacional, em que se observou maior rapidez de execução na posição ventral 23,24 .

Com relação às perdas sanguíneas no intraoperatório, analisadas pela diferença da taxa de hemoglobina e hematócrito no pré e pós-operatório, não foi observada diferença estatisticamente significante entre as duas posições. Na literatura a posição ventral é associada a menor perda sanguínea em relação à de Lloyd-Davies ${ }^{24}$. Transfusões sanguíneas ocorreram em 37,8\% dos pacientes do grupo Lloyd-Davis e em 15,8\% dos pacientes do grupo ventral, apesar de não ter diferença estatisticamente significante, tais dados foram semelhantes a estudos internacionais ${ }^{24}$.
A diferença de volume intravenoso recebido no intraoperatório foi estatisticamente significante entre os grupos, com o grupo da posição ventral tendo recebido menor quantidade.

As complicações perioperatórias, as precoces e os óbitos não tiveram diferença estatisticamente significante entre os grupos, em contraste com o melhor desempenho da posição ventral nesse aspecto na literatura ${ }^{24}$. A análise das complicações tardias de um modo geral foi estatisticamente significante, com uma maior predominância delas no grupo Lloyd-Davies, concordando com a literatura pesquisada ${ }^{24}$. Quanto à duração da internação, não se observou significância estatística entre os grupos, mas na literatura a posição ventral foi relacionada a um menor tempo de internação ${ }^{24}$.

As margens circunferenciais das peças, assim como a variação da quantidade de linfonodos retirados nas peças cirúrgicas dos dois grupos também não tiveram significância estatística, bem como a recidiva local e sistêmica. Em alguns estudos a recidiva local apresentou menor incidência no grupo ventral, e em outros, essa diferença não foi identificada ${ }^{10,23}$. A análise do tempo livre de doença mostrou-se sem diferença estatisticamente significante entre os grupos, fato também observado em um estudo chinês de $2015^{24}$.

Concluímos que a análise dos fatores relacionados às diferentes posições para realização da cirurgia de amputação abdominoperineal de reto revelou que o grupo da posição ventral obteve menor necessidade de infusão de volume intravenoso durante a cirurgia e uma menor incidência de complicações pós-operatórias tardias, enquanto que o grupo da posição de Lloyd-Davies obteve melhores tempos cirúrgicos e anestésicos. Não foram observadas diferenças estatisticamente significantes entre os dois grupos nos quesitos sangramento e taxa de transfusão sanguínea no intraoperatório, complicações perioperatórias e recentes, óbitos, duração da internação, comprometimento da margem circunferencial e número de linfonodos 
dissecados. A avaliação da recidiva, tempo livre de doença e sobrevida global será realizada após maior tempo de seguimento.

Em comparação com a posição LloydDavies, a posição ventral parece ser a melhor escolha para os pacientes submetidos à amputação abdominoperineal do reto devido ao menor índice de complicações tardias. No entanto, maior tempo de seguimento é necessário para realizar a escolha da posição cirúrgica baseada em desfecho oncológico.

\section{A B S T R A C T}

Objective: to evaluate the benefits and disadvantages of the ventral decubitus position compared with that of LloydDavies in patients submitted to abdominoperineal amputation of the rectum. Methods: we conducted a retrospective study of 56 patients submitted to abdominoperineal amputation of the rectum due to distal rectal and anal canal neoplasms, treated at the Central Hospital of the Santa Casa de Misericórdia in São Paulo between 2008 and 2017. Results: patients' mean age was 63.08 years, $48.2 \%$ of them women and $51.8 \%$, men. Adenocarcinoma was the histological type, in $94.6 \%$ of cases, and squamous cell carcinoma, in 5.4\%. The position of Lloyd-Davies was adopted in $66.1 \%$ of the procedures, and the ventral position, in $33.9 \%$. At the time of surgery, four patients had synchronous metastases: hepatic (one case), pulmonary (one case) and simultaneous liver and lung (two cases). Neoadjuvant treatment was performed in $85.7 \%$ of the patients. Late postoperative complications occurred in 13 patients operated in the classic position and in one patient operated on in the ventral decubitus position. The overall survival time for the group operated in the classic position was on average 45.7 months, while in the group operated on in the ventral decubitus position it was 15.5 months. Conclusion: the ventral position group presented less need for intraoperative intravenous volume infusion and fewer postoperative complications, whereas the Lloyd-Davies group had better surgical and anesthetic times. Relapse, disease-free time, and overall survival should be evaluated at a longer follow-up time.

Keywords: Intestinal Neoplasms. Amputation. Rectal Neoplasms. Colorectal Surgery.

\section{REFERÊNCIAS}

1. Instituto Nacional de Câncer José Alencar Gomes da Silva. Coordenação de Prevenção e Vigilância Estimativa 2014: Incidência de Câncer no Brasil. Rio de Janeiro: INCA, 2014. Disponível em: \&lt;http://www.inca.gov.br/estimativa/2014/index. asp?|D=7\&gt;

2. Hamilton SR, Aaltonen LA. Pathology and Genetics of Tumours of the Digestive System. Lion: IARC 2000. Disponível em: <http://www.iarc.fr/en/ publications/pdfs-online/pat-gen/bb2/BB2.pdf>

3. Stewart SL, Wike JM, Kato I, Lewis DR, Michaud F. A population-based study of colorectal cancer histology in the United States, 1998-2001. Cancer. 2006;107(5 Suppl):1128-41.

4. Schmidt CE, Bestmann B, Küchler T, Longo WE, Kremer B. Ten-year historic cohort of quality of life and sexuality in patients with rectal cancer. Dis Colon Rectum. 2005;48(3):483-92.

5. Cruz GMG, Ferreira RMRS, Neves PM. Amputação abdominoperineal: uma cirurgia fora de moda? Estudo retrospectivo de 135 cirurgias realizadas ao longo de quatro décadas. Rev Bras Coloproct. 2004;24(2):103-18.
6. Burgos FJ, Romero J, Fernández E, Perales L, Tallada M. Risk factor for developing voiding dysfunction after abdominoperineal resection for adenocarcinoma of the rectum. Dis Colon Rectum. 1988;31(9):682-7.

7. Bokey E, Chapuis PH, Hughes WJ, Joorey SG. Morbidity, mortality and survival following resection for carcinoma of the rectum of Concord Hospital. Aust N Z J Surg. 1990;60(4):253-9.

8. Lloyd-Davies OV. Lithotomy-Trendelenburg position for resection of rectum and lower pelvic colon. Lancet. 1939;234(6045):74-6.

9. Edgecombe $H$, Carter K, Yarrow S. Anaesthesia in the prone position. Br J Anaesth. 2008;100(2):165-83.

10. Tayyab M, Sharma A, Ragg JL, Macdonald AW, Gunn J, Hartley JE, et al. Evaluation of the impact of implementing the prone jackknife position for the perineal phase of abdominoperineal excision of the rectum. Dis Colon Rectum. 2012;55(3):316-21.

11. Jemal A, Siegel $R, X u$ J, Ward E. Cancer Statistics, 2010. CA Cancer J Clin. 2010;60(5):277-300. Erratum in: CA Cancer J Clin. 2011;61(2):133-4.

12. Ferlay J, Soerjomataram I, Ervik M, Dikshit R, Eser S, Mathers C, et al. GLOBOCAN 2012 v1.0, Cancer Incidence and Mortality Worldwide: 
IARC CancerBase No. 11 [Internet]. Lyon, France: International Agency for Research on Cancer; 2013. Disponível em:\&lt; http://globocan.iarc.fr\&gt;

13. Murphy G, Devesa SS, Cross AJ, Inskip PD, McGlynn $K A$, Cook MB. Sex disparities in colorectal cancer incidence by anatomic subsite, race and age. Int J Cancer. 2010;128(7):1668-75.

14. American Cancer Society. Colorectal Cancer Facts \& Figures 2014-2016. Atlanta: American Cancer Society; 2014. Disponível em: \&lt;http:// www.cancer.org/acs/groups/content/documents/ document/acspc-042280.pdf \&gt;

15. Howlader N, Noone AM, Krapcho M, Garshell J, Neyman N, Alekruse SF, editors, et al. SEER Cancer Statistics Review, 1975-2010. Bethesda, MD: National Cancer Institute; 2013.

16. Larsson SC, Orsini N, Wolk A. Diabetes mellitus and risk of colorectal cancer: a meta-analysis. J Natl Cancer Inst. 2005;97(22):1679-87.

17. Campbell PT, Deka A, Jacobs EJ, Newton CC, Hildebrand JS, McCullough ML, et al. Prospective study reveals associations between colorectal cancer and type 2 diabetes mellitus or insulin use in men. Gastroenterology. 2010;139(4):113846.

18. Secretan B, Straif $K$, Baan R, Grosse $Y, E l$ Guissassi F, Bouvard V, Benbrahim-Tallaa L, Guha N, Freeman C, Galichet L, Cogliano V; WHO International Agency for Research on Cancer Monograph Working Group. A review of human carcinogens - Part E: tobacco, areca nut, alcohol, coal smoke, and salted fish. Lancet Oncol. 2009;10(11):1033-4.

19. Liang PS, Chen TY, Giovannucci E. Cigarette smoking and colorectal cancer incidence and mortality: systematic review and meta-analysis. Int J Cancer. 2009;124(10):2406-15.

20. Paskett ED, Reeves KW, Rohan TE, Allison MA, Williams CD, Messina CR, et al. Association between cigarette smoking and colorectal cancer in the Women's Health Initiative. J Natl Cancer Inst. 2007;99(22):1729-35.

21. Giovannucci E. An updated review of the epidemiological evidence that cigarette smoking increases risk of colorectal cancer. Cancer Epidemiol Biomarkers Prev. 2001;10(7):725-31.

22. Limsui $D$, Vierkant RA, Tillmans LS, Wang $A H$, Weisenberger DJ, Laird PW, et al. Cigarette smoking and colorectal cancer risk by molecularly defined subtypes. J Natl Cancer Inst. 2010;102(14):1012-22.

23. Hu X, Cao L, Zhang J, Liang P, Liu G. Therapeutic results of abdominoperineal resection in the prone jackknife position for T3-4 low rectal cancers. J Gastrointest Surg. 2015;19(3):551-7.

24. Liu P, Bao H, Zhang X, Zhang J, Ma L, Wang Y, et al. Better operative outcomes achieved with the prone jackknife vs. lithotomy position during abdominoperineal resection in patients with low rectal cancer. World J Surg Oncol. 2015;13:39.

Recebido em: 18/06/2018

Aceito para publicação em: 08/08/2018

Conflito de interesse: nenhum.

Fonte de financiamento: nenhuma.

\section{Endereço para correspondência:}

Rodrigo Barbosa Novais

E-mail: digoal@gmail.com

caue.fedrigo@gmail.com

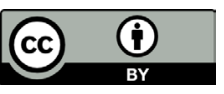

